\title{
Downward-Looking Linear Array 3D SAR Imaging Based on Multiple Measurement Vectors Model and Continuous Compressive Sensing
}

\author{
Qi-yong Liu, ${ }^{1,2}$ Qun Zhang, ${ }^{1,2,3}$ Fu-fei Gu, ${ }^{4}$ Yi-chang Chen, ${ }^{1,2}$ Le Kang, ${ }^{1,2}$ and Xiao-yu Qu ${ }^{1}$ \\ ${ }^{1}$ Information and Navigation College, Air Force Engineering University, Xian 710077, China \\ ${ }^{2}$ Collaborative Innovation Center of Information Sensing and Understanding, Xian 710077, China \\ ${ }^{3}$ Key Laboratory for Information Science of Electromagnetic Waves, Fudan University, Shanghai 200433, China \\ ${ }^{4}$ China Satellite Maritime Tracking and Control Department, Jiangyin 214431, China
}

Correspondence should be addressed to Qun Zhang; zhangqunnus@gmail.com

Received 17 January 2017; Accepted 20 March 2017; Published 5 April 2017

Academic Editor: Stephane Evoy

Copyright (C) 2017 Qi-yong Liu et al. This is an open access article distributed under the Creative Commons Attribution License, which permits unrestricted use, distribution, and reproduction in any medium, provided the original work is properly cited.

\begin{abstract}
This paper concerns the problems of huge data and off-grid effect of cross-track direction in downward-looking linear array (DLLA) 3D SAR imaging. Since the 3D imaging needs a great deal of memory space, we consider the methods of downsampling to reduce the data quantity. In the azimuth direction, we proposed a method based on the multiple measurement vectors (MMV) model, which can enhance computational efficiency and elevate the performance of antinoise, to recover the signal. Further, in crosstrack direction, since the resolution is restricted by the length of array, as well as platform size, the influence of off-grid effect is more serious than azimuth direction. Continuous compressive sensing (CCS), which can solve the off-grid effect of the classical compressive sensing (CS), is presented to obtain the precise imaging result under the noise scenarios. Finally, we validate our method by extension numerical experiments.
\end{abstract}

\section{Introduction}

Downward-looking linear array three-dimensional synthetic aperture radar (DLLA 3D SAR) can not only overcome the restrictions of shading and layover effects [1,2], but also keep the advantages of conventional SAR. In DLLA 3D imaging, the height resolution can be acquired by transmitting big bandwidth signal, and the azimuth (along-track) resolution can be acquired by synthetic aperture formed by platform movement, and the cross-track resolution can be acquired by linear array along cross-track direction [3, 4]. DLLA 3D SAR has obtained attention abroad in recent years. Many scholars have extended the conventional SAR imaging algorithms into DLLA 3D imaging, for example, 3D chirp scaling algorithm [5], 3D range migration algorithm [6], and 3D polar format algorithm [7].

To obtain superresolution imaging result, Chen and Zhang introduced the multiple signal classification (MUSIC) algorithm in cross-track direction and azimuth direction processing [8]. Furthermore, Zhang et al. combined the fast Fourier transform and MUSIC (FFT-MUSIC) to promote the computational efficiency [9].

In practical, the $3 \mathrm{D}$ observed scene behaves with typical sparsity in a certain domain. Sparse signal recovery and compressive sensing (CS) methods provide a solution to 3D imaging algorithm. Zhang et al. produced 2D SL0 algorithm for signal reconstruction on azimuth and cross-track direction [10]. But, in the classical model of CS, the sparse signal is recovered from a single measurement vector, which is called single measurement vector (SMV) model [11]. In DLLA 3D imaging, linear array can get multi-SMV along azimuth. Meanwhile, each SMV signal has the same sparse structure. We consider the problem of finding sparse representation of signals in azimuth direction from multiple measurement vectors, which is known as the MMV model [12]. Compared to SMV model, MMV model can enhance the computational efficiency and elevate the antinoise performance. 
Actually, within the classical CS framework, the signal has to be represented with a finite discrete dictionary. However signals in applications such as radar, array processing, and communication are usually specified by parameters in a continuous domain. In order to apply the theory of CS to these applications, researchers have to adopt a discretization procedure to reduce the continuous parameter space to a finite set of grid points $[13,14]$, which otherwise will cause the off-grid effect $[15,16]$. Due to the poor resolution of crosstrack direction, the off-grid effect has a greater impact in cross-track direction than azimuth direction.

To solve the off-grid effect, many methods based on sparse representations have been proposed [17, 18]. But these methods are also based on the discretization processing. Based on atomic norm, atomic norm minimization approach is proposed [19]. By reformulating the atomic norm minimization (ANM) as a semidefinite programming (SDP) problem, the signal can be recovered from continuous dictionary instead of discrete dictionary. This method is also called continuous compressive sensing (CCS). CCS has been applied to line spectral estimation and direction of arrival (DOA) estimation to solve the off-grid problem [20-22]. Furthermore, Bao et al. introduce the CCS to DLLA 3D imaging with pseudo-polar format [23, 24].

In this paper, we exploit MMV model and CCS method for DLLA 3D SAR imaging at azimuth and cross-track direction. Firstly, if we want high azimuth resolution, the azimuth synthetic aperture is also very long, which will cause huge sampling number. Thus we proposed a method based on MMV model to recover azimuth signal. Compared to SMV model, MMV model will enhance the computational efficiency and elevate the performance of antinoise. Furthermore, in cross-track, the resolution is restricted by the length of array, as well as platform size; the influence of off-grid effect is more serious than azimuth direction. So we utilize CCS method, which can solve the off-grid effect of CS, to obtain the precise imaging result under the noise scenarios. Finally, we validate our theory by extension numerical experiments.

The rest of this paper is organized as follows. Section 2 formulates the signal model of DLLA 3D SAR. Section 3 builds the MMV model along cross-track to reconstruct the azimuth signal. In Section 4, the CCS method is presented to reconstruct cross-track signal. Simulation experiments are carried out in Section 5. Finally, some conclusions are drawn in Section 6.

\section{DLLA 3D SAR Imaging Modeling}

The imaging geometry of DLLA 3D SAR using linear array is shown in Figure 1. The plane is supposed to fly at the altitude $H$ along the flight path (azimuth direction) of the platform $(X$-axis) with the velocity $v$. The linear array is composed of $N$ antenna elements, which are mounted underneath the wings along the cross-track direction ( $Y$-axis) and symmetrical about $Z$-axis, and equally spaced with the distance $d$. The $Z$ axis denotes the height direction (range direction). Assuming $N$ is odd number, the $n$th antenna element is located at

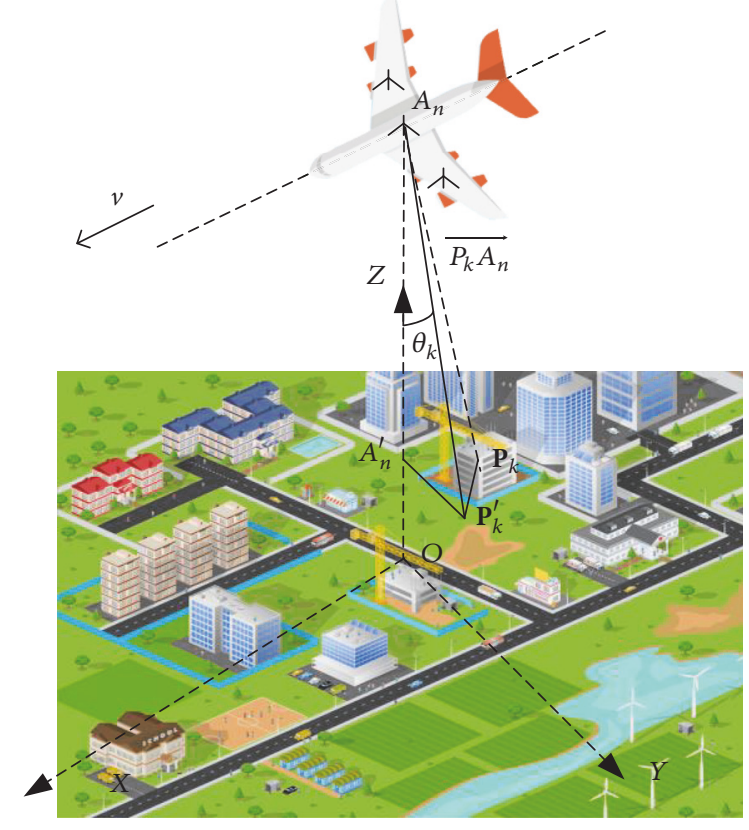

FIGURE 1: DLLA 3D SAR imaging geometry model.

$A_{n}\left(x_{m}, y_{n}, H\right)$ at slow time $t_{m}$, where $x_{m}=v \cdot t_{m}, y_{n}=(n-(N-$ 1)/2) $\cdot d . A_{n} A_{n}^{\prime} \perp X O Y . \perp$ is the vertical symbol. Additionally, the linear array of $N$ elements can also be obtained with sparse MIMO array by equivalent phase center principle [25]. In this paper, we do not consider the wings oscillations, which will be studied in the future. The form of single-transmission and multireception of linear array is utilized to obtain the signal. We assume that the movement compensation has been accomplished before the image reconstruction. Actually, the platform movement during the airborne DLLA 3D SAR array transmission and reception can be compensated before image reconstruction $[26,27]$. The following equations have no account of the platform movement during array transmission and reception.

Assuming there are $K$ scatterers, each scatterer is located at $P_{k}\left(x_{k}, y_{k}, z_{k}\right), k=1,2, \ldots, K . P_{k}^{\prime}$ is its projection onto $Y O Z$ plane. The distance between $P_{k}$ and the $n$th antenna $A_{n}$ at slow time $t_{m}$ is $R_{P A}\left(t_{m} ; k, n\right)=\left\|\overrightarrow{P_{k} A_{n}}\right\|$. Assuming the radar transmits a linear frequency modulation (LFM) signal with the center frequency $f_{c}$, on the condition of far field, the received acquisition data of the $n$th antenna element can be expressed as

$$
\begin{aligned}
s_{n}\left(\widehat{t}, t_{m}\right)= & \sum_{k=1}^{K} \sigma_{k} \exp \left(-j 2 \pi f_{c} \frac{2 \cdot R_{P A}\left(t_{m} ; k, n\right)}{c}\right) \\
& \cdot \exp \left(j \pi K_{r}\left(\hat{t}-\frac{2 \cdot R_{P A}\left(t_{m} ; k, n\right)}{c}\right)^{2}\right),
\end{aligned}
$$

where $\sigma_{k}$ is the reflectance, $\hat{t}$ is the fast time, $c$ is the electromagnetic wave speed, and $K_{r}$ is the chirp rate. The 
instantaneous distance $R_{P A}\left(t_{m} ; k, n\right)$ can be approximately expressed as

$$
R_{P A}\left(t_{m} ; k, n\right) \approx R_{B}+\frac{\left(v t_{m}-x_{k}\right)^{2}}{2 R_{B}}+\frac{y_{n}^{2}-2 y_{n} y_{k}}{2 R_{B}},
$$

where $R_{B}=\sqrt{\left(H-z_{k}\right)^{2}+y_{k}^{2}}$.

Once the raw data have been focused into a "Sinc" function by the range compression, the echo signal can be reduced into a $2 \mathrm{D}$ problem of the azimuth and cross-track direction. Then, the echo signal can be rewritten as [10]

$$
\begin{aligned}
s_{n}\left(t_{m}\right)= & \sum_{k=1}^{K} \zeta_{k} \exp \left(-j \frac{2 \pi}{\lambda} \frac{\left(v t_{m}-x_{k}\right)^{2}}{R_{B}}\right) \\
& \cdot \exp \left(-j \frac{2 \pi}{\lambda} \frac{y_{n}^{2}-2 y_{n} y_{k}}{R_{B}}\right),
\end{aligned}
$$

where $\lambda=c / f_{c}$ is wavelength, $\zeta_{k} \approx C \cdot \operatorname{sinc}\left(\Delta f_{r} \cdot\left(\widehat{t}-\left(2 R_{B} / c\right)\right)\right)$ reflects the reflectance of the point targets, $C$ is a constant value, and $\Delta f_{r}$ is the bandwidth of transmitted signal. Then, a deramping operation is performed to remove the independent quadratic phase terms of cross-track direction in echo signal. Therefore, echo signal can be rewritten as

$$
\begin{aligned}
s_{n}\left(t_{m}\right)= & \sum_{k=1}^{K} \zeta_{k} \exp \left(-j \frac{2 \pi}{\lambda} \frac{\left(v t_{m}-x_{k}\right)^{2}}{R_{B}}\right) \\
& \cdot \exp \left(j 2 \pi \frac{2 y_{n} y_{k}}{\lambda R_{B}}\right) .
\end{aligned}
$$

In (4), the first exponent term is only related to azimuth direction. The second exponent term is only related to crosstrack direction. Thus we can handle the azimuth direction and cross-track direction, respectively. The structure of crosstrack direction cannot be damaged in azimuth processing.

\section{MMV Modeling for Azimuth Reconstruction}

Firstly, sparsity is a prerequisite for CS. In DLLA 3D SAR imaging, there are large amounts of nontarget zones in the different elevations of the 3D scene, which renders the signals sparse on the azimuth and cross-track plane [28]. That is, the elevation signal to be reconstructed is sparse in the object domain; that is, only a few scatterers with different azimuths and cross-tracks are presented in the same range cell [24].

Here, a brief review of CS theory will be given.

3.1. CS Reconstruction. The signal $\mathbf{x}=\left[x(1) ; x(2) ; \ldots ; x\left(N_{1}\right)\right] \epsilon$ $\mathbb{C}^{N_{1} \times 1}$ is represented if there is an orthogonal basis $\Psi=$ $\left\{\psi_{1}, \psi_{2}, \ldots, \psi_{N}\right\} \in \mathbb{C}^{N_{1} \times N}$ as follows:

$$
\mathbf{x}=\sum_{k=1}^{N} \psi_{k} \alpha_{k}=\mathbf{\Psi} \boldsymbol{\alpha}
$$

where $\boldsymbol{\alpha} \in \mathbb{C}^{N \times 1}$ is the coefficient sequence of $\mathbf{x}$ related to basis $\Psi$ and $\alpha_{k}=\left\langle\mathbf{x}, \psi_{k}\right\rangle$ is the inner product of $\mathbf{x}$ and $\psi_{k} . \boldsymbol{\alpha}$ is said to be $K$-sparse if it has, at most, $K$ nonzero elements (or absolute value comparatively big [29]). $\Psi$ is the sparse dictionary. The sparse signal $\mathbf{x}$ can be recovered by the low-dimensional measurements of $\mathbf{x}$ through CS theory. One important merit of CS is to recover the sparse signal from the fewest possible measurements. By collecting the measurement vectors as rows of a sensing matrix $\Phi \in \mathbb{R}^{M \times N_{1}}$, and $M \ll N_{1}$, in the presence of measurement noise e, the acquisition procedure can be succinctly written as

$$
\mathrm{y}=\Phi \mathbf{x}+\mathrm{e}=\Phi \Psi \alpha+\mathrm{e} .
$$

Within the CS framework, if the mapping matrices $\boldsymbol{\Theta}=\boldsymbol{\Phi} \boldsymbol{\Psi}$ fulfill certain requirements, for example, the restricted incoherence property (RIP) and the incoherence property, $\mathbf{x}$ can be accurately recovered from its compressive measurements with a high probability by solving the problem

$$
\begin{aligned}
\widehat{\boldsymbol{\alpha}}=\min _{\boldsymbol{\alpha}} & \|\boldsymbol{\alpha}\|_{1}, \\
\text { s.t. } & \|\mathbf{y}-\boldsymbol{\Theta} \boldsymbol{\alpha}\|_{2}<\varepsilon,
\end{aligned}
$$

where $\varepsilon$ is an upper bound on the $l_{2}$ norm of the noise vector e and $\|\cdot\|_{1}$ denotes the $l_{1}$ norm, which is equal to the sum of the absolute values of the vector entries. The downsample rate (DSR) is defined as DSR $=M / N_{1}$.

3.2. Reconstruction with MMV Model. Firstly, with the CS framework, we should construct the sparse dictionary of azimuth. After the range compression, the azimuth signal can be recovered for each range cell in turn. According to the theory of matched filtering, the azimuth compression function $H_{a}\left(f_{a}\right)$ can be expressed as

$$
H_{a}\left(f_{a}\right)=\exp \left(j \frac{\pi}{\gamma} f_{a}^{2}\right),
$$

where $\gamma=-2 v^{2} /\left(\lambda R_{B}\right)$.

Thus the azimuth focusing can be implemented by the following operation:

$$
g_{n}\left(t_{m}\right)=\operatorname{IFFT}\left\{H_{a} \cdot \operatorname{FFT}\left[s_{n}\left(t_{m}\right)\right]\right\} .
$$

After azimuth compression, the signal is sparse in azimuth. Thus we can recover the azimuth signal with lowdimensional measurement signal by the CS method. Assuming $\mathbf{s}_{n}=\left[s_{n}\left(t_{1}\right) ; \ldots ; s_{n}\left(t_{M_{1}}\right)\right] \in \mathbb{C}^{M_{1} \times 1}$ is the measurement signal of $n$th antenna with $M_{1}$ sample number in azimuth direction, $\mathbf{g}_{n}=\left[g_{n}\left(t_{1}\right) ; \ldots ; g_{n}\left(t_{M_{1}}\right)\right] \in \mathbb{C}^{M_{1} \times 1}$ is the corresponding focused signal after azimuth focusing.

According to the procedure of matched filtering, the azimuth compression operation of $n$th antenna signal can be rewritten as matrix expression

$$
\mathbf{g}_{n}=\Psi_{2} \cdot \operatorname{diag}\left(H_{a}\right) \cdot \Psi_{1} \cdot \mathbf{s}_{n}=\Psi_{a} \cdot \mathbf{s}_{n} \quad n=1, \ldots, N,
$$

where $\Psi_{1} \in \mathbb{C}^{M_{1} \times M_{1}}$ denotes DFT matrices. $\Psi_{2} \in \mathbb{C}^{M_{1} \times M_{1}}$ denotes IDFT matrices. $\operatorname{diag}\left(H_{a}\right) \in \mathbb{C}^{M_{1} \times M_{1}}$ denotes the diagonalization operation of compression function $H_{a}\left(f_{a}\right)$. $\Psi_{a}=\Psi_{2} \cdot \operatorname{diag}\left(H_{a}\right) \cdot \Psi_{1}$ is the matched filtering matrices. 
Through simple transformation, the above equation can be expressed as

$$
\mathbf{s}_{n}=\Psi_{a}^{-1} \cdot \mathbf{g}_{n} \quad n=1, \ldots, N
$$

where $\Psi_{a}^{-1}$ is the inverse matrix of $\Psi_{a}$.

The structure of (11) is coincident with the CS expression. So we can recover the azimuth signal $\mathbf{g}_{n}$ from measurement vector $\mathbf{s}_{n}$ with CS theory. $\Psi_{a}^{-1}$ is the sparse dictionary.

Next, we can get the low-dimensional measurement vector through downsampling. We choose the random partial unit matrix as sensing matrix $\Phi_{n} \in \mathbb{R}^{M \times M_{1}}$. So the downsampling signal can be expressed as

$$
\mathbf{s}_{n}^{\prime}=\Phi_{n} \mathbf{s}_{n}=\Phi_{n} \Psi_{a}^{-1} \cdot \mathbf{g}_{n} \quad n=1, \ldots, N,
$$

where $\mathbf{s}_{n}^{\prime}$ is the downsampling signal.

As is discussed in [30], the randomly selected partial orthogonal matrix satisfies RIP. $\boldsymbol{\Phi}_{n}$ is a random partial unit matrix. So if the sparse dictionary $\Psi_{a}^{-1}$ is an orthogonal matrix, $\boldsymbol{\Phi}_{n} \Psi_{a}^{-1}$ obeys the RIP. Thus, for azimuth measurement vector, the sparse representation $\mathbf{g}_{n}$ of signal $\mathbf{s}_{n}$ can be recovered by the following problem:

$$
\begin{aligned}
\widehat{\mathbf{g}}_{n}=\min _{\mathbf{g}_{n}} & \left\|\mathbf{g}_{n}\right\|_{1}, \\
& \text { s.t. }\left\|\mathbf{s}_{n}^{\prime}-\boldsymbol{\Phi}_{n} \boldsymbol{\Psi}_{a}^{-1} \mathbf{g}_{n}\right\|_{2}<\varepsilon .
\end{aligned}
$$

The above analysis is based on single measurement vector, which is also called SMV model. For the linear array, each antenna element can obtain a single measurement vector. Normally, with $N$ times SMV model, we can get the azimuth recovery signal of linear array. But the computational efficiency of this approach is very low. We can utilize the MMV model to elevate the computational efficiency. In the case of MMV model, the sparse signals of all elements should share the same sparse structure. This condition is easy to satisfy for the multiple measurement vectors signal of linear array.

In the reconstruction of azimuth signal, the cross-track direction is vertical with azimuth direction. Meanwhile, at the far field condition, with the length restriction of array antenna, there is no range migration to the same target for all antenna elements. It implies that the sparse structure of each measurement is the same. Thus the azimuth direction recovery can be implemented by MMV model. Additionally, we can also adopt the same sensing matrix $\Phi_{a} \in \mathbb{R}^{M \times M_{1}}$ for the multiple measurement vectors. That is, we can take the $\Phi_{n}$ as $\Phi_{a}$ for $n=1, \ldots, N$.

Thus the multiple measurement vectors can be denoted as $\mathbf{S}=\left[\mathbf{s}_{1}, \ldots, \mathbf{s}_{N}\right] \in \mathbb{C}^{M_{1} \times N}$. The downsampling signal can be denoted as $\boldsymbol{\Xi}=\boldsymbol{\Phi}_{a} \mathbf{S}$. The recovery signal can be denoted as $\mathbf{G}=\left[\mathbf{g}_{1}, \ldots, \mathbf{g}_{N}\right] \in \mathbb{C}^{M_{1} \times N}$. So the problem with MMV model can be reformulated as follows:

$$
\begin{aligned}
\widehat{\mathbf{G}}=\min _{\mathbf{G}} & \|\mathbf{G}\|_{2,1} \\
\text { s.t. } & \left\|\boldsymbol{E}-\boldsymbol{\Phi}_{a} \Psi_{a}^{-1} \mathbf{G}\right\|_{2}<\varepsilon,
\end{aligned}
$$

where $\varepsilon \in \mathbb{C}^{M \times N} \cdot\|\cdot\|_{2,1}$ is the $(2,1)$ norm which is defined by $\|\mathbf{G}\|_{2,1}=\sum_{i=1}^{N}\left\|\mathbf{G}^{i}\right\|_{2}$ and $\mathbf{G}^{i}$ is the $i$ th row of $\mathbf{G}$.
In this paper, we adopt the method of MMV-OMP, which is an extension of Orthogonal Matching Pursuit (OMP) method, to deal with the MMV model.

When the azimuth signal recovery for all range cells is completed, the signal can be reformulated as

$$
g_{n}=\sum_{k=1}^{K} \rho_{k} \exp \left(j 2 \pi \frac{2 y_{n} y_{k}}{\lambda R_{B}}\right)
$$

where $\rho_{k} \approx C^{\prime} \cdot \operatorname{sinc}\left(\Delta f_{r} \cdot\left(\widehat{t}-\left(2 R_{B} / c\right)\right)\right) \cdot \operatorname{sinc}\left(\Delta f_{a} \cdot\left(t_{m}-\left(x_{k} / v\right)\right)\right)$ reflects the reflectance of the point targets, $C^{\prime}$ is a constant value, and $\Delta f_{a}$ is the Doppler bandwidth.

Here, the range compression and reconstruction of azimuth direction are completed. Only cross-track direction is left to be reconstructed in following steps. With the length limitation of linear array, the resolution of cross-track direction is poor. To obtain precise location of cross-track, we adopt the CCS method, which can eliminate the off-grid effect and recover the cross-track signal.

\section{Continuous Compressed Sensing (CCS) for Cross-Track Reconstruction}

As we all know, in the framework of traditional CS, we need to adopt a discretization procedure to reduce the continuous parameter space to a finite set of grid points. Discretization has several significant drawbacks. Firstly, the true parameters do not fall onto the finite grid, which is called off-grid effect. On the other hand, the dictionary becomes very coherent, which will lead to instability of the signal reconstruction, as we increase the number of grid points. Moreover, finer grids will also produce huge computational burden [19].

4.1. Transformation of Cross-Track Signal. Generally, the spacing $d$ of antenna element can take half a wavelength $\lambda$, for example, $d=\lambda / 2$. Recall that the cross-track coordinate of $n$th antenna element is $y_{n}=(n-(N-1) / 2) \cdot d$, and $N$ is odd number. Actually, the cross-track signal recovery is completed for each range cell and azimuth cell in turn. Assume the number of scatterers is $K^{\prime}$ in pixel $\left(r_{e}, a_{e}\right)$, where $r_{e}$ represents range cell and $a_{e}$ represents the azimuth cell. So (15) can be rewritten as

$$
g_{n}=\sum_{k=1}^{K^{\prime}} \rho_{k} \exp \left(j \cdot 2 \pi \frac{y_{k}}{R_{B}} \cdot\left(n-\frac{(N-1)}{2}\right)\right) .
$$

Define $h_{k}=y_{k} / R_{B}=\sin \left(\theta_{k}\right)$, where $\theta_{k}$ is the inclination angle between $\overrightarrow{A_{n} A_{n}^{\prime}}$ and $\overrightarrow{A_{n} P_{k}}$ projection onto YOZ plane. To satisfy unambiguous imaging conditions of DLLA 3D SAR imaging, the beam width is limited by $|\theta| \leq 15^{\circ}$ [7], which is the subset of $\theta \in[-\pi / 6, \pi / 6]$. So $h_{k}=\sin (\theta) \in[-1 / 2,1 / 2]$. Define $n^{\prime}=n-(N-1) / 2$. So $n^{\prime}=-(N-1) / 2, \ldots,(N-1) / 2$. In 
the following description, we will use the notation $n$ to replace $n^{\prime}$. Thus (16) can be simplified as

$$
\begin{aligned}
g_{n}=\sum_{k=1}^{K^{\prime}} \rho_{k} \exp \left(j \cdot 2 \pi h_{k} \cdot n\right) & \\
n & =-\frac{(N-1)}{2}, \ldots, \frac{(N-1)}{2},
\end{aligned}
$$

where $\rho_{k}$ is a complex number. It can be described as $\rho_{k}=$ $\left|\rho_{k}\right| \exp \left(j \varphi_{k}\right) . \varphi_{k} \in[0,2 \pi)$ is the corresponding phase of $\rho_{k}$. We can define index set $\mathbf{D}=-(N-1) / 2, \ldots,(N-1) / 2$. Apparently, the expression is in accordance with the expression of atomic norm $[19,20]$. In the case of atomic norm, the frequencies $f$ must afford the condition of $f \in[0,1]$, and $f$ can take continuous values. That is, $h_{k}$ must belong to $[0,1]$. So we can take some simple transformation with a trivial translation of the frequency domain. Multiplying both sides of (17) with exponential term $\exp (j \pi \cdot n)$ and considering the noise, we have

$$
\begin{array}{r}
g_{n}^{\prime}=g_{n} \exp (j \pi \cdot n)=\sum_{k=1}^{K^{\prime}} \rho_{k} \exp \left(j \cdot 2 \pi f_{k} \cdot n\right)+w_{n} \\
n \in \mathbf{D},
\end{array}
$$

where $f_{k}=y_{k} / R_{B}+1 / 2$, and $f_{k} \in[0,1] . w_{n}$ is the noise. In the rest of the paper, we use $\Omega=\mathbf{f}=\left\{f_{1}, f_{2}, \ldots, f_{K^{\prime}}\right\} \subset[0,1]$ to denote the unknown set of frequencies. Then, we can use atomic norm to solve this problem.

4.2. Atomic Norm Minimization (ANM). Equation (18) is full sampling form. We can also sample the signal at a subset of the linear array. Assume the index set $T \subset[-(N-1) / 2, \ldots,(N-$ 1)/2] of size $M_{T}$ is selected uniformly from $\mathbf{D}$ at random. In the following description, we will use notation $g_{n}$ to replace $g_{n}^{\prime}$. So the observation signal can be expressed as

$$
g_{n}^{\dagger}=\sum_{k=1}^{K^{\prime}} \rho_{k} \exp \left(j \cdot 2 \pi f_{k} \cdot n\right)+w_{n}, \quad n \in T \subset \mathbf{D} .
$$

The above equation can be reformulated as

$$
\mathbf{g}^{\dagger}=\sum_{k=1}^{K^{\prime}}\left|\rho_{k}\right| \mathbf{a}\left(f_{k}, \varphi_{k}\right)+\mathbf{w}
$$

where $\left[a\left(f_{k}, \varphi_{k}\right)\right]_{n}=\exp \left(j\left(2 \pi f_{k} \cdot n+\varphi_{k}\right)\right)$. So the atoms can be defined as $a(f, \varphi) \in \mathbb{C}^{|T|} ;|\cdot|$ is the element number of set, $f \in[0,1], \varphi \in[0,2 \pi)$. The set of atoms $\mathscr{A}$ are building blocks of the signal $\mathbf{g}^{\dagger}$, with $\mathscr{A}=\{a(f, \phi): f \in[0,1], \phi \in[0,2 \pi)\}$. Then atomic norm is defined as the gauge function of the hull $\mathscr{A}[19]$ :

$$
\begin{aligned}
\|\mathbf{g}\|_{\mathscr{A}} & =\inf \{t>0: \mathbf{g} \in t \cdot \operatorname{conv}(\mathscr{A})\} \\
& =\inf _{\substack{\rho_{k} \geq 0, f_{k} \in[0,1], \varphi_{k} \in[0,2 \pi)}}\left\{\sum_{k}\left|\rho_{k}\right|: \mathbf{g}=\sum_{k}\left|\rho_{k}\right| a\left(f_{k}, \varphi_{k}\right)\right\} .
\end{aligned}
$$

With the idea of using atomic norm to enforce sparsity for a general set of atoms [30], the atomic norm $\|\cdot\|_{\mathscr{A}}$ can enforce sparsity in $\mathscr{A}$ because low-dimensional faces of $\operatorname{conv}(\mathscr{A})$ correspond to signals involving only a few atoms [16].

Recall that we observe only a subset of entries $\mathbf{T} \subset \mathbf{D}$. We can use atomic norm minimization (ANM) problem to estimate the missing samples of a sparse sum of complex exponentials.

$$
\begin{array}{ll}
\min _{\mathbf{g}} & \|\mathbf{g}\|_{\mathscr{A}} \\
\text { s.t. } & \left\|\mathbf{g}_{T}-\mathbf{g}_{T}^{\dagger}\right\|_{2}<\varepsilon,
\end{array}
$$

where $\varepsilon$ denotes the noise level.

4.3. Reconstruction with CCS. Through the Vandermonde decomposition lemma, the problem of ANM can be equivalent to semidefinite program (SDP) problem. The semidefinite program problem of $(22)$ is

$$
\begin{array}{ll}
\min _{\mathbf{u}, \mathbf{t}, \mathbf{g}} & \frac{1}{2|\mathbf{D}|} \operatorname{trace}(\operatorname{Toep}(\mathbf{u}))+\frac{1}{2} \mathbf{t} \\
\text { s.t. } & {\left[\begin{array}{cc}
\text { Toep }(\mathbf{u}) & \mathbf{g} \\
\mathbf{g}^{*} & \mathbf{t}
\end{array}\right] \geq 0} \\
& \left\|\mathbf{g}_{T}-\mathbf{g}_{T}^{\dagger}\right\|_{2}<\varepsilon,
\end{array}
$$

where trance $(\cdot)$ is the sum of main diagonal elements. The superscript “*” denotes conjugate transpose and Toep $(\mathbf{u})$ denotes the Toeplitz matrix whose first column is equal to $\mathbf{u}$. $\mathbf{u}=\sum_{k}\left|\rho_{k}\right| a\left(f_{k}, 0\right)$ and $\mathbf{t}=\sum_{k}\left|\rho_{k}\right|$. This semidefinite program always recovers the missing samples and identifies the frequencies provided the number of measurements is large enough and the frequencies are reasonably well separated. The SDPs can be solved using a standard SDP solver, SDPT3 [31]. The relationship between reconstruction probability and sampling number is analyzed in the paper [23]. When the minimum separable normalized frequency satisfies

$$
\Delta f=\min _{i \neq j}\left|f_{i}-f_{j}\right| \geq \frac{c_{1}}{\lfloor(N-1) / 4\rfloor},
$$

for some small universal constant $c_{1}$, then there exists a numerical constant $C$ so that

$$
M_{T} \geq C \max \left\{\log ^{2} \frac{N}{\varsigma}, K^{\prime} \log ^{2} \frac{K^{\prime}}{\varsigma} \log ^{2} \frac{N}{\varsigma}\right\}
$$

is enough to reconstruct the scatterers in cross-track direction with probability at least $1-\varsigma$. In the case of nonuniform samples, this restriction may break.

The dual problem [19] of (23) can be expressed as

$$
\begin{array}{cl}
\max _{\mathbf{q}} & \left\langle\mathbf{q}_{T}, \mathbf{g}_{T}^{\dagger}\right\rangle_{\mathbb{R}} \\
\text { s.t. } & \|\mathbf{q}\|_{\mathscr{A}}^{*} \leq 1 \\
& \mathbf{q}_{T^{c}}=0,
\end{array}
$$




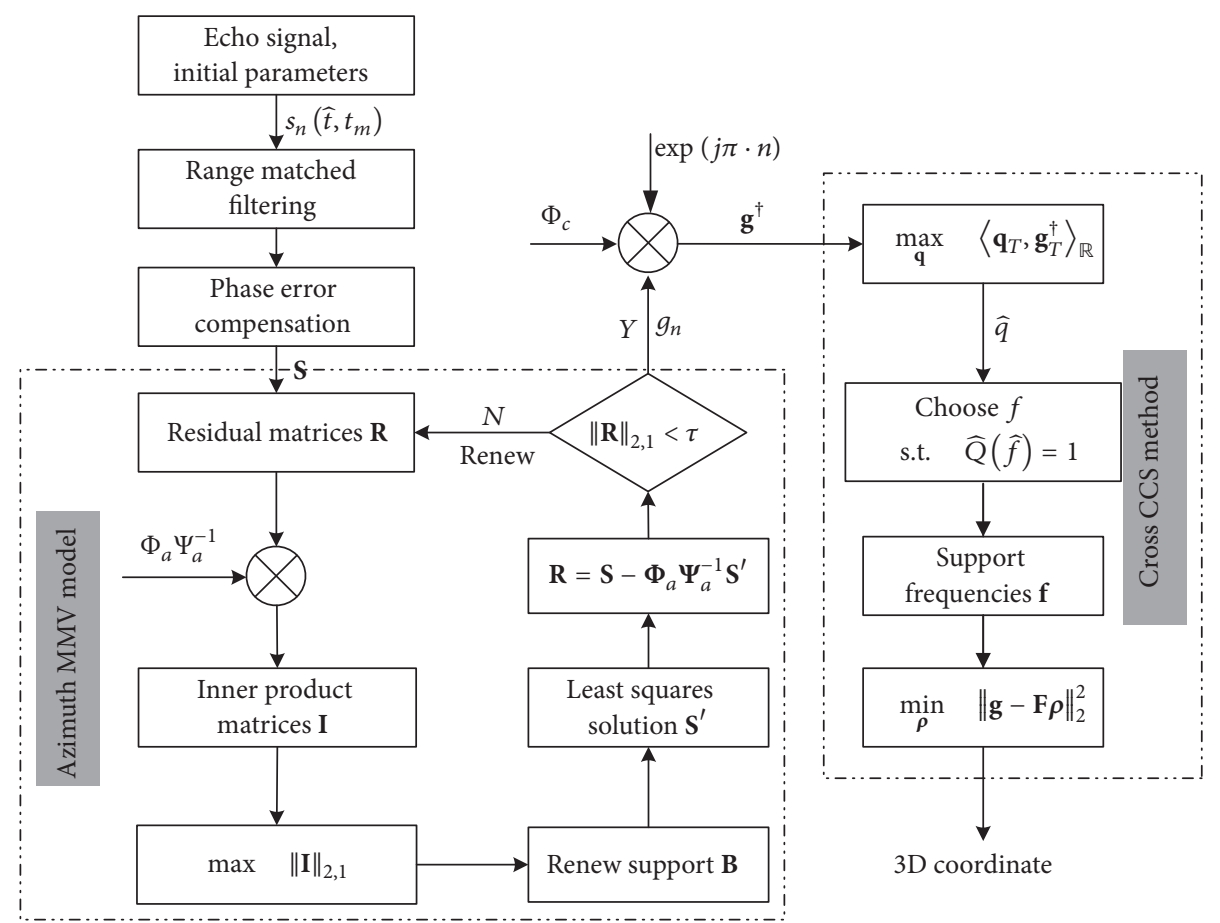

FIGURE 2: The flowchart of DLLA 3D SAR imaging.

where $\|\mathbf{q}\|_{\mathscr{A}}^{*}=\sup _{\mathbf{a} \in \mathscr{A}}\langle\mathbf{q}, \mathbf{a}\rangle_{\mathbb{R}}$ is the dual norm of $\|\cdot\|_{\mathscr{A}}$; $\langle\mathbf{q}, \mathbf{g}\rangle_{\mathbb{R}}=\operatorname{Re}(\langle\mathbf{q}, \mathbf{g}\rangle)$ is the real inner product. Once the dual solution $\widehat{q}$ is obtained, the frequency support set that can be located by $\widehat{Q}(f)$ achieves modulus 1 , and $\widehat{Q}(f):=$ $\langle\widehat{q}, a(f, 0)\rangle=\sum_{d \in D} q_{d} \exp (-j 2 \pi f \cdot d)$. The evaluation of $\widehat{Q}(f)$ can perform efficiently using fast Fourier transform (FFT). Once the frequencies are estimated, the coefficients can be obtained by solving the following problem:

$$
\begin{array}{ll}
\min _{\boldsymbol{\rho}} & \|\mathbf{g}-\mathbf{F} \boldsymbol{\rho}\|_{2}^{2} \\
\text { s.t. } & \mathbf{F}_{d k}=\exp \left(j 2 \pi f_{k} \cdot d\right) .
\end{array}
$$

We can use cross-track direction sensing matrix $\boldsymbol{\Phi}_{c} \in$ $\mathbb{R}^{M \times N}$ to select the array element. $\Phi_{c}$ is a random partial unit matrix. Through analyzing, we can know that (23) can adopt the CCS for processing. Then we can get $f_{k}$ and further $y_{k}$ by solving the SDP problem. Finally, we can get the scatters' amplitude $\rho_{k}$ through least square method.

The basis flowchart of DLLA 3D SAR imaging with MMV model in azimuth direction and CCS in cross-track direction is shown in Figure 2.

\section{Numerical Experiments}

We conducted a series of numerical experiments to test the performance of the proposed algorithm. The parameters of platform and antenna, which are referenced to the ARTINO system [4], are shown in Table 1. The locations and amplitude parameters of scatterers are shown in Table 2.
TABle 1: Parameters of platform and antenna.

\begin{tabular}{lc}
\hline Parameters & Value \\
\hline Carrier frequency $f_{c}(\mathrm{GHz})$ & 37.5 \\
Bandwidth $B_{r}(\mathrm{MHz})$ & 300 \\
Height of platform $H(\mathrm{~m})$ & 500 \\
Velocity of platform $v(\mathrm{~m} / \mathrm{s})$ & 15 \\
Pulse duration $T_{r}(\mathrm{us})$ & 0.1 \\
Pulse repeat frequency, PRF $(\mathrm{Hz})$ & 1000 \\
Space distance of adjacent EPC $d(\mathrm{~m})$ & 0.004 \\
Number of transmitting antennas $N_{t}$ & 10 \\
Number of receiving antennas $N_{r}$ & 11 \\
Cross-track resolution $(\mathrm{m})$ & 4.5 \\
Azimuth resolution $(\mathrm{m})$ & 0.5 \\
\hline
\end{tabular}

TABLE 2: Parameters of scatterers.

\begin{tabular}{lcccc}
\hline \multicolumn{2}{l}{ Scatterers Azimuth $(\mathrm{m})$} & Height $(\mathrm{m})$ & Cross-track $(\mathrm{m})$ & Amplitude \\
\hline 1 & -20 & 0.8 & -10.3 & 1 \\
2 & -20 & 0.8 & 9.4 & 1 \\
3 & -20 & 12.4 & -4.5 & 1 \\
4 & -20 & 12.3 & 4.2 & 1 \\
5 & 20 & 2.82 & -9.3 & 1 \\
6 & 20 & 2.8 & 9.4 & 1 \\
7 & 10 & 12.4 & -4.5 & 1 \\
8 & 10 & 12.3 & 4.2 & 1 \\
9 & 0 & 5 & 10.5 & 1 \\
\hline
\end{tabular}




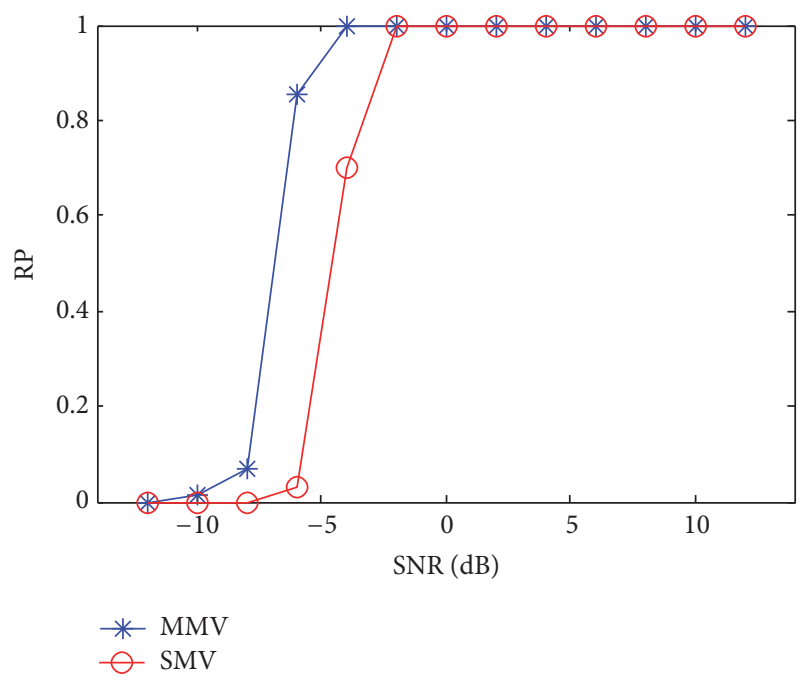

FIgURE 3: The relationship between SNR and Recovery Probability (RP).

5.1. The Performance Analysis of MMV Model. After range compression, signal is composed of azimuth and cross-track plane. So we can deal with the range cell in turn. In azimuth and cross-track plane, each antenna element can obtain a measurement signal along azimuth. The linear array can obtain $N$ measurement signal. We can adopt the MMV model for recovery azimuth signal.

To verify that the performance of MMV model outperforms the performance of SMV model, we firstly take the Monte Carlo trials in different signal-to-noise ratio (SNR) to compare the Recovery Probability (RP). The time of Monte Carlo trials is 100 . The scope of SNR is $-12 \mathrm{~dB} \sim 12 \mathrm{~dB}$ with $2 \mathrm{~dB}$ step. The downsample rate (DSR) is 0.5 . The relationship between SNR and RP is shown in Figure 3.

Figure 3 implies SNR is smaller than $-14 \mathrm{~dB}$; both SMV and MMV model cannot obtain the exact solution caused by the noise. With the increase of SNR, the RP is also elevated. Also, the RP of MMV model is increased more quickly than the SMV model. When the SNR reaches $-10 \mathrm{~dB}$, the RP of MMV model starts to increase. When the SNR reaches $-4 \mathrm{~dB}$, the RP reaches 1 . However the RP of SMV model starts to increase when the SNR reaches $-8 \mathrm{~dB}$. Until the SNR reaches $-2 \mathrm{~dB}$, the RP of SMV model reaches 1 . In other words, compared to SMV model, MMV model can boost the antinoise ability.

Then, we obtained the imaging results when DSR is 0.5 and $\mathrm{SNR}$ is $-6 \mathrm{~dB}$. In order to reflect the antinoise performance of MMV model, the cross-track signal reconstruction is completed with OMP algorithm. There are nine scatterers shown in Table 2 for simulation. The imaging results are shown in Figure 4. Figures 4(a) and 4(b) show the reconstruction results without cross-track reconstruction, where the result of Figure 4(a) is reconstructed with MMV model in azimuth direction and the result of Figure 4(b) is reconstructed with SMV model in azimuth direction, respectively. It can be found that the azimuth signal is almost submerged by the noise in Figure 4(b). Figures 4(c) and 4(d) show the reconstruction results after accomplishing the three-dimensional reconstruction to Figures 4(a) and 4(b) with OMP algorithm, respectively. Comparing Figures 4(c) and $4(\mathrm{~d})$, we can see that there are two scatterers that have been missed in Figure 4(d). It verifies that the MMV model can elevate the antinoise performance.

Moreover, we take the Monte Carlo trials in different DSR to compare the RP. The SNR is $-6 \mathrm{~dB}$. Figure 5 shows the relationship between DSR and RP. When the DSR is low, the $\mathrm{RP}$ is very low in both MMV and SMV model. With the increase of DSR, the RP is also elevated in both MMV and SMV model. But the RP of MMV model increases quicker than the SMV model. That is, with the same SNR and DSR, the recovery accuracy of MMV model is better than the SMV model. Actually, the DSR can decrease further in MMV model.

Furthermore, we have finished the reconstruction of azimuth with different $L$ of MMV model and different DSR. The relationship between reconstruction time of azimuth and DSR is shown in Figure 6. Assuming there is a target. We only reconstruct azimuth signal of the range cell, which contains the target. The sample number of azimuth is 420 . The number of antenna elements is 110 . So the size of raw data is $420 \times$ 110. The SNR is $0 \mathrm{~dB}$. The reconstruction time is the average time of 150 Monte Carlo trials. The result shows, with the DSR increase, that the reconstruction time also rises under the same $L$. With the increase of $L$, the reconstruction time is decreased under the same DSR. Thus MMV model can increase the computational efficiency.

5.2. Continuous CS (CCS). In the framework of traditional $\mathrm{CS}$, there is off-grid effect which causes the recovery result to be imprecise. CCS can solve the off-grid effect through solving the SDP problem which is equivalent to ANM instead of discretization frequency domain.

To verify the performance of CCS, we carried out some trials with L1-norm method of conventional CS and CCS method. The L1-norm is solved by CVX package. To highlight the precise estimation performance of CCS to L1norm method, we conduct some quantitative comparisons. The normalized frequencies and amplitude are $(0.165,1)$, $(0.334,1)$, and $(0.56,0.78)$, respectively. The scope of sparse dictionary is $0 \sim 1$ with 0.02 steps. The sampling number $N$ is 20. Downsample number $M$ is 15 . The DSR is $M / N=$ 0.75 . Figure 4 shows the reconstruction results of normalized frequency.

Figure 7 shows that the L1-norm can estimate the accurate frequency which locates on the grid, for example, the third frequency. When the frequency does not locate on the grid, there are some errors, for example, the first and second frequency. Only those frequencies, which locate on the grid, can be reconstructed in accuracy with L1-norm. If the frequency does not locate on the grid, the frequency will be projected to the dictionary. Actually, L1-norm chooses the maximum correlation dictionary to replace the target frequency. Usually, the frequency may fission into two frequencies. Namely, the accuracy of both frequencies and amplitude estimation of the CCS method are better than the L1-norm method. 


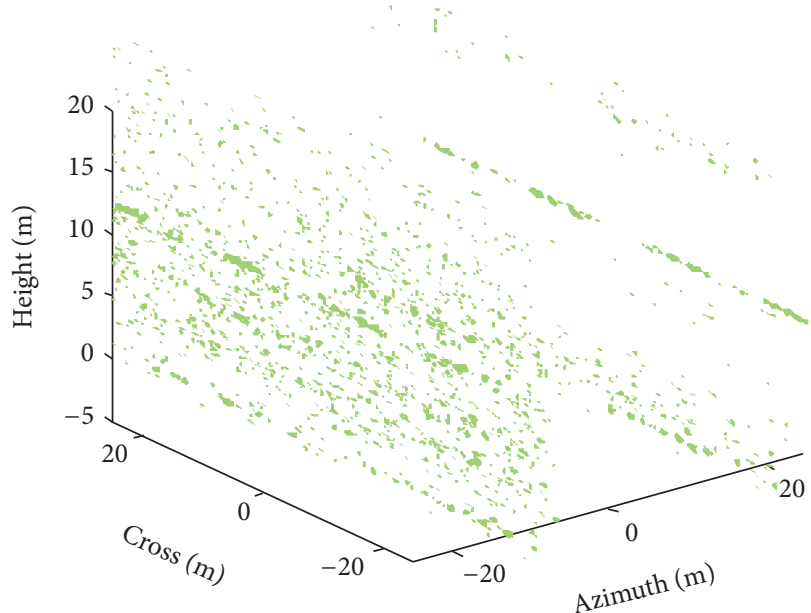

(a)

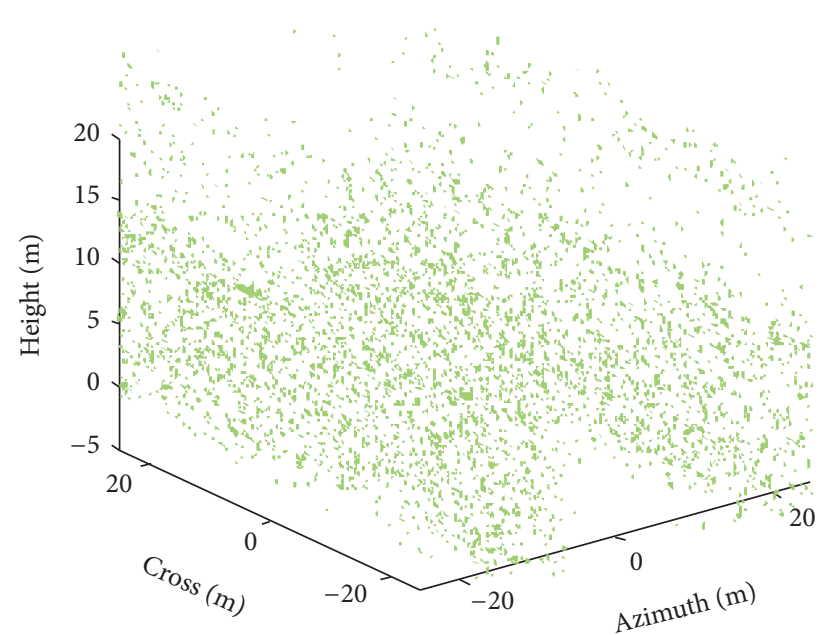

(b)

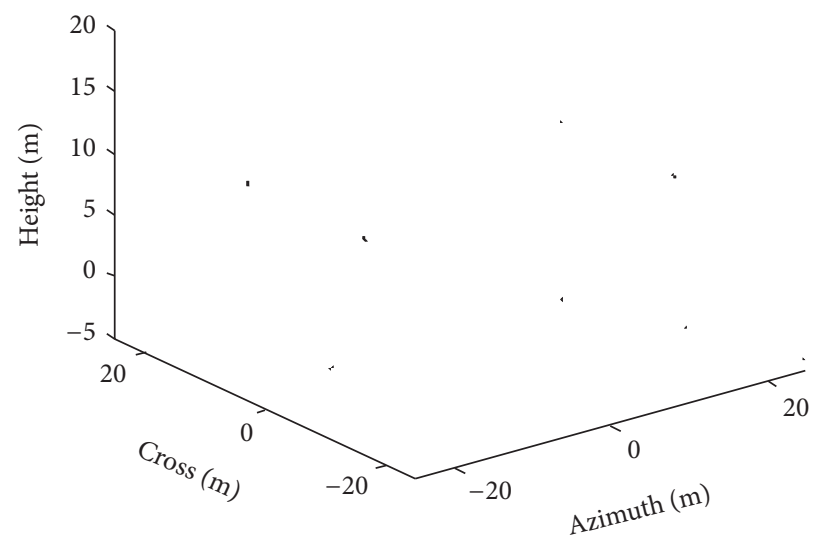

(d)

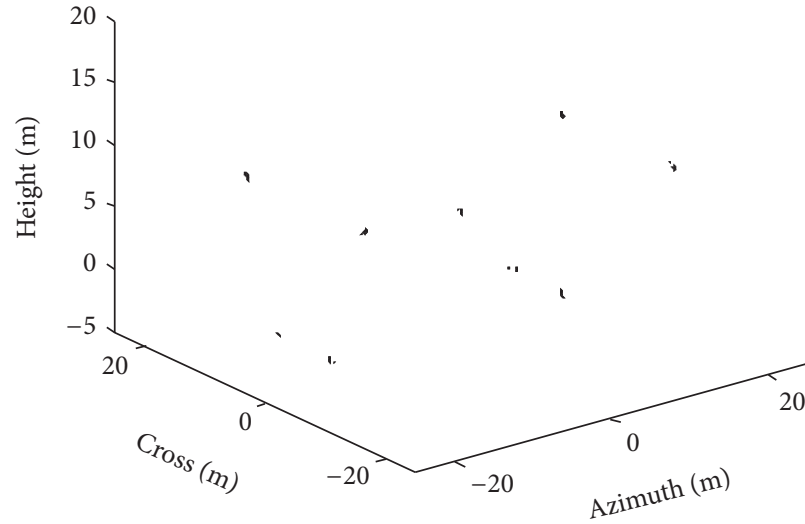

(c)

Figure 4: Three-dimensional imagery. (a) Completed the range matched filtering and azimuth MMV reconstruction; (b) completed the range matched filtering and azimuth SMV reconstruction; (c) completed the range matched filtering and azimuth MMV reconstruction and crosstrack SMV reconstruction; (d) completed the range matched filtering and azimuth SMV reconstruction and cross-track SMV reconstruction.

5.3. CCS of Cross-Track Direction. We randomly choose $70 \%$ antenna arrays from the filled virtual array and the SNR is $5 \mathrm{~dB}$ of raw echo data. The DSR of azimuth is 0.5 . There are nine scatterers with the unit reflectivity in the Cartesian coordinate system, as shown in Figure 8(a).

Assume the grid spacing is chosen as $\rho_{c}=\gamma \cdot D_{c}$ at crosstrack, where $D_{c}$ is the Rayleigh resolution of the cross-track direction. $\gamma$ is the dictionary factor of cross-track and $\gamma=$ 0.05 . Figure $8(\mathrm{a})$ also shows the $3 \mathrm{D}$ imaging reconstruction resulting from the proposed algorithm. Figure $8(\mathrm{~b})$ shows reconstruction resulting from the $L 1$-norm method. There are some ghost scatterers in Figure 8(b).

Figure 9(a) shows the projection onto azimuth and cross-track plane of true scatterers, reconstructed by proposed method and L1-norm method. Figure 9(b) shows the projection onto cross-track direction. It shows that the ANM method can obtain more accurate value than the L1norm method. The L1-norm method suffered the off-grid effect.
TABLE 3: Performances comparisons of CCS and L1-norm method.

\begin{tabular}{lccccc}
\hline \multirow{2}{*}{ Performances } & \multirow{2}{*}{ CCS } & \multicolumn{5}{c}{ Dictionary factor $\gamma$} \\
& & 0.01 & 0.02 & 0.05 & 0.1 \\
\hline RMSE (m) & 0.0502 & 0.0576 & 0.0601 & 0.0612 & 0.0859 \\
Mean running time (s) & 93.65 & 236.14 & 107.199 & 45.58 & 27.65 \\
\hline
\end{tabular}

Further, we changed the sparse factor as $\gamma=0.01, \gamma=0.05$, and $\gamma=0.1$ in cross-track, respectively. The SNR is $10 \mathrm{~dB}$. The mean value of root mean square error (RMSE) and the mean running time of single trial are shown in Table 3 of cross-track direction with 100 Monte Carlo trials. With the decrease of sparse factor $\gamma$, the dictionary becomes finer and the RMSE also decreases. But the running time increases. When the sparse factor is $\gamma=0.02$, the running time of $L 1$-norm method exceeded the CCS method. But the RMSE of CCS is less than the MRMSE of L1-norm. This is because the L1-norm suffers 


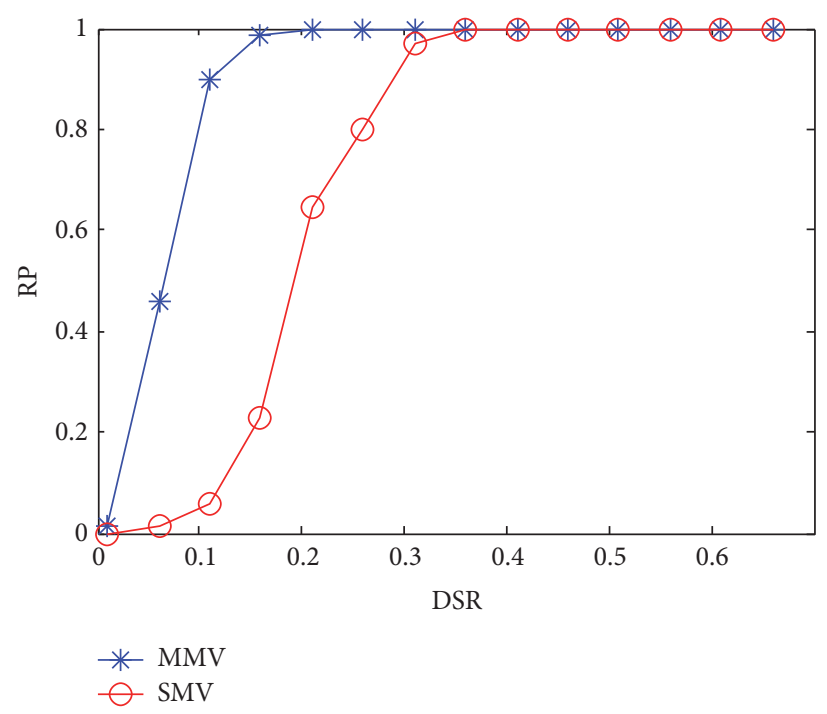

FIgURE 5: The relationship between DSR and Recovery Probability (RP).

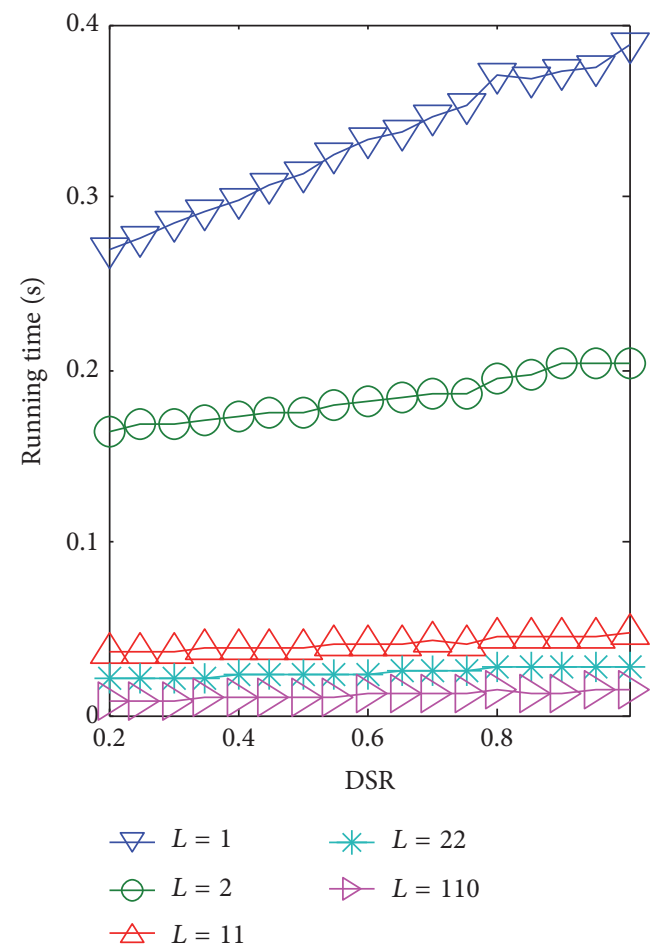

FIGURE 6: The relationship between reconstruction time and DSR.

the off-grid effect, which causes the estimation result to be imprecise. That is, the CCS can eliminate the off-grid effect.

\section{Conclusions}

In this paper, we exploit the MMV model and the CCS method for DLLA 3D SAR imaging at azimuth and crosstrack direction. With the application of MMV model in azimuth, the data quantity can decrease further and the computational efficiency is more outstanding. On the other hand,

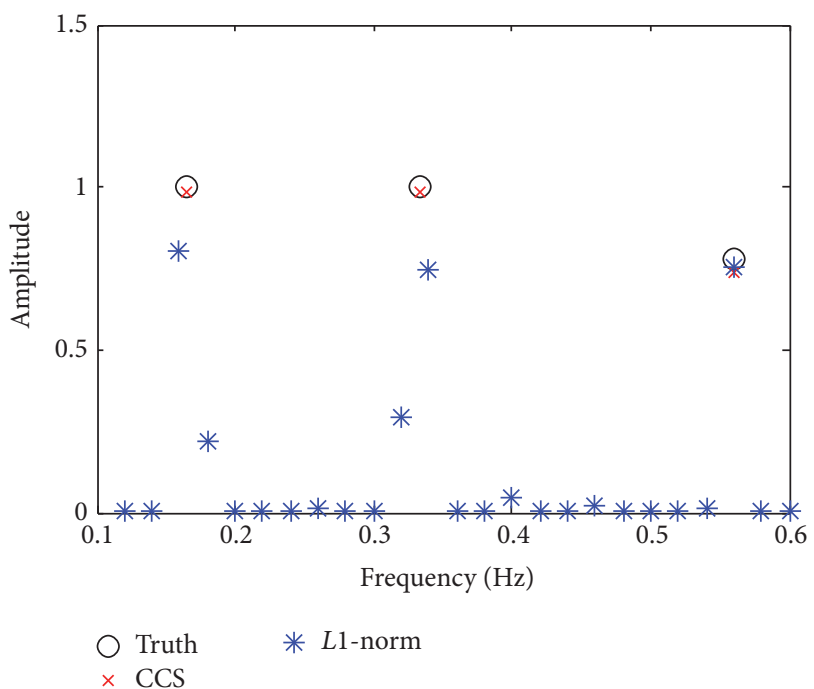

FIGURE 7: Reconstruction results of normalized frequency.

it can elevate the performance of antinoise. Further, in crosstrack direction, since the resolution is restricted by the length of array, as well as platform size, the influence of off-grid effect is more serious than azimuth direction. We utilize CCS, which can also solve the off-grid effect of CS, to reconstruct the cross-track signal under the noise scenarios. Finally, we validate our theory by extension numerical experiments. As the CCS method is more sensitive than the L1-norm about noise, in the following researches, we will investigate the application of CCS in low SNR.

\section{Conflicts of Interest}

The authors declare that there are no conflicts of interest regarding the publication of this paper. 


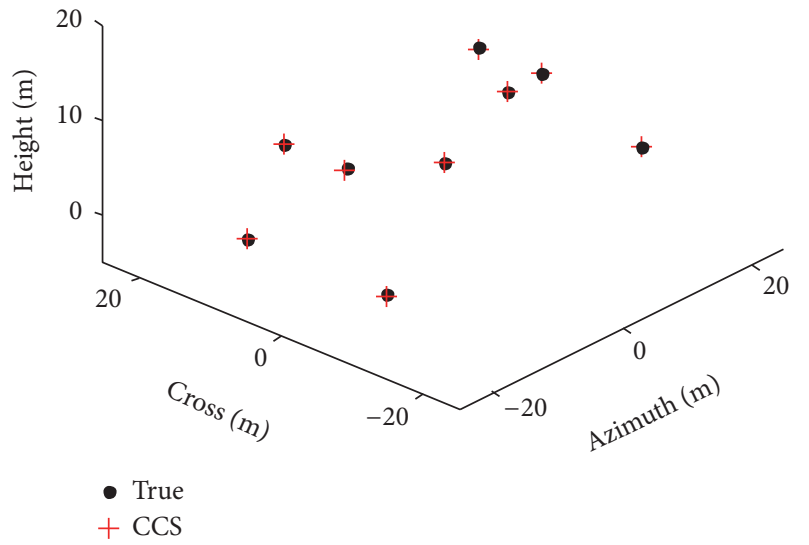

(a)

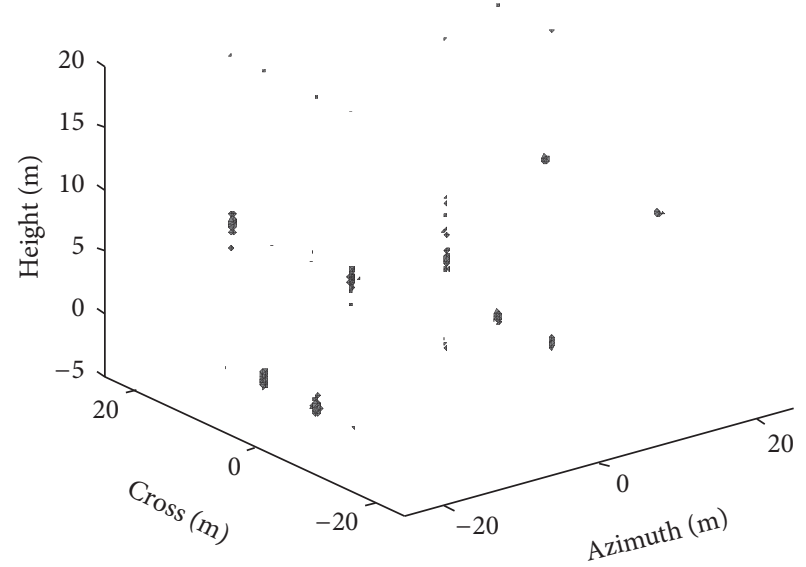

(b)

FIGURE 8: True scatterers and reconstruction result. (a) True location and reconstruction of proposed method; (b) reconstruction of $L 1$-norm.

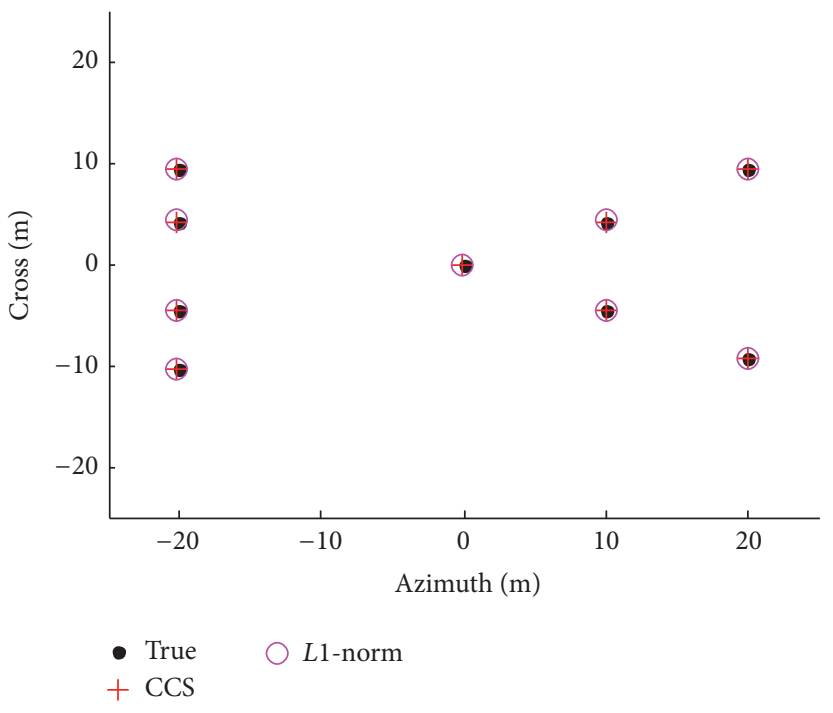

(a)

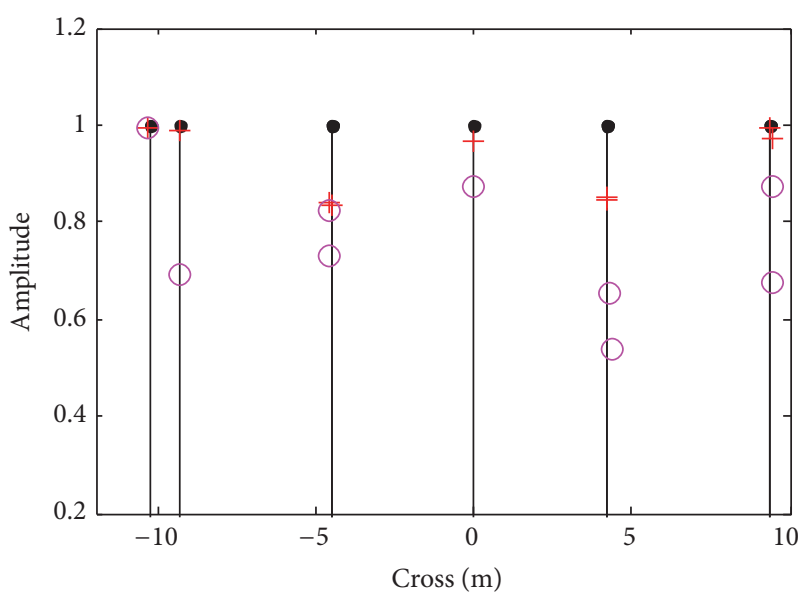

(b)

Figure 9: The projection of true scatterers and reconstruction result. (a) Projection on azimuth and cross plane; (b) projection on cross-track.

\section{Acknowledgments}

This work was supported in part by the National Natural Science Foundation of China under Grants 61501498 and 61631019.

\section{References}

[1] M. Soumekh, Synthetic Aperture Radar Signal Processing with MATLAB Algorithms, Wiley, New York, NY, USA, 1999.

[2] X. Wencheng, Z. Xiaoling, and S. Jun, "MIMO antenna array design for airborne down-looking 3D imaging SAR," in Proceedings of the 2nd International Conference on Signal Processing Systems (ICSPS '10), pp. V2-452-V2-456, IEEE, Dalian, China, July 2010.
[3] J. F. Nouvel, H. Jeuland, G. Bonin, S. Roques, O. Du Plessis, and J. Peyret, "A Ka band imaging radar: DRIVE on board ONERA motorglider," in Proceedings of the IEEE International Geoscience and Remote Sensing Symposium (IGARSS '06), pp. 134-136, Denver, Colo, USA, August 2006.

[4] M. Weib and J. Ender, "A 3-D imaging radar for small unmanned airplanes-ARTINO," in Proceedings of the European Radar Conference, pp. 209-212, Paris, France, 2005.

[5] D. H. Zhang and X. L. Zhang, "Downward-looking 3-D linear array SAR imaging based on chirp scaling algorithm," in Proceedings of the 2nd Asian-Pacific Conference on Synthetic Aperture Radar (APSAR '09), pp. 1043-1046, IEEE, Xi'an, China, October 2009.

[6] L. Du, Y. P. Wang, W. Hong, W. Tan, and Y. Wu, "A threedimensional range migration algorithm for downward-looking 3-D SAR with single-transmitting and multiple-receiving linear 
array antennas," EURASIP Journal on Advances in Signal Processing, vol. 2010, Article ID 957916, 15 pages, 2010.

[7] X. Peng, W. Hong, Y. Wang, W. Tan, and Y. Wu, "Polar format imaging algorithm with wave-front curvature phase error compensation for airborne DLSLA three-dimensional SAR," IEEE Geoscience and Remote Sensing Letters, vol. 11, no. 6, pp. 1036-1040, 2014.

[8] C. Chen and X. Zhang, "A newsuper-resolution 3-D SAR imaging method based on MUSIC algorithm," in Proceedings of the RADAR Conference, pp. 525-529, Kansas, Mo, USA, May 2011.

[9] S. Q. Zhang, Y. T. Zhu, and G. Y. Kuang, "Imaging of downwardlooking linear array three-dimensional SAR Based on FFTMUSIC," IEEE Geoscience and Remote Sensing Letters, vol. 12, no. 4, pp. 885-889, 2015.

[10] S. Zhang, G. Dong, and G. Kuang, "Superresolution downwardlooking linear array three-dimensional SAR imaging based on two-dimensional compressive sensing," IEEE Journal of Selected Topics in Applied Earth Observations and Remote Sensing, vol. 9, no. 6, pp. 2184-2196, 2016.

[11] E. J. Candes, J. Romberg, and T. Tao, "Robust uncertainty principles: exact signal reconstruction from highly incomplete frequency information," IEEE Transactions on Information Theory, vol. 52, no. 2, pp. 489-509, 2006.

[12] J. Chen and X. Huo, "Theoretical results on sparse representations of multiple-measurement vectors," IEEE Transactions on Signal Processing, vol. 54, no. 12, pp. 4634-4643, 2006.

[13] R. Baraniuk and P. Steeghs, "Compressive radar imaging," in Proceedings of the IEEE Radar Conference, pp. 128-133, IEEE, Waltham, Mass, USA, April 2007.

[14] X. X. Zhu and R. Bamler, "Tomographic SAR inversion by L1norm regularization - the compressive sensing approach," IEEE Transactions on Geoscience and Remote Sensing, vol. 48, no. 10, pp. 3839-3846, 2010.

[15] Y. Chi, L. L. Scharf, A. Pezeshki, and A. R. Calderbank, "Sensitivity to basis mismatch in compressed sensing," IEEE Transactions on Signal Processing, vol. 59, no. 5, pp. 2182-2195, 2011.

[16] M. A. Herman and T. Strohmer, "General deviants: an analysis of perturbations in compressed sensing," IEEE Journal on Selected Topics in Signal Processing, vol. 4, no. 2, pp. 342-349, 2010.

[17] M. F. Duarte and R. G. Baraniuk, "Spectral compressive sensing," Applied and Computational Harmonic Analysis, vol. 35, no. 1, pp. 111-129, 2013.

[18] Y. Chi, L. L. Scharf, A. Pezeshki, and A. R. Calderbank, "Sensitivity to basis mismatch in compressed sensing," IEEE Transactions on Signal Processing, vol. 59, no. 5, pp. 2182-2195, 2011.

[19] G. Tang, B. N. Bhaskar, P. Shah, and B. Recht, "Compressed sensing off the grid," IEEE Transactions on Information Theory, vol. 59, no. 11, pp. 7465-7490, 2013.

[20] Z. Yang and L. Xie, "Enhancing sparsity and resolution via reweighted atomic norm minimization," IEEE Transactions on Signal Processing, vol. 64, no. 4, pp. 995-1006, 2016.

[21] Z. Yang and L. Xie, "Continuous compressed sensing with a single or multiple measurement vectors," in Proceedings of the IEEE Workshop on Statistical Signal Processing (SSP '14), pp. 288-291, IEEE, Gold Coast, Australia, July 2014.

[22] B. N. Bhaskar, G. Tang, and B. Recht, "Atomic norm denoising with applications to line spectral estimation," IEEE Transactions on Signal Processing, vol. 61, no. 23, pp. 5987-5999, 2013.
[23] Q. Bao, K. Han, X. Peng, W. Hong, B. Zhang, and W. Tan, "DLSLA 3-D SAR imaging algorithm for off-grid targets based on pseudo-polar formatting and atomic norm minimization," Science China Information Sciences, vol. 59, no. 6, Article ID $062310,2016$.

[24] Q. Bao, K. Han, Y. Lin, B. Zhang, J. Liu, and W. Hong, "Imaging method for downward-looking sparse linear array threedimensional synthetic aperture radar based on reweighted atomic norm," Journal of Applied Remote Sensing, vol. 10, no. 1, Article ID 015008, 2016.

[25] J. Klare, A New Airborne Radar for 3D Imaging-Simulation Study of ARTION, EURAR, Dresden, Germany, 2006.

[26] X. Peng, W. Tan, W. Hong, C. Jiang, Q. Bao, and Y. Wang, "Airborne DLSLA 3-D SAR image reconstruction by combination of polar formatting and L1 regularization," IEEE Transactions on Geoscience and Remote Sensing, vol. 54, no. 1, pp. 213-226, 2016.

[27] A. Meta, P. Hoogeboom, and L. P. Ligthart, "Correction of the effects induced by the continuous motion in airborne FMCW SAR," in Proceedings of the IEEE Radar Conference, pp. 358-365, Verona, NY, USA, April 2006.

[28] S.-J. Wei, X.-L. Zhang, and J. Shi, "Linear array SAR imaging via compressed sensing," Progress in Electromagnetics Research, vol. 117, pp. 299-319, 2011.

[29] M. F. Duarte and R. G. Baraniuk, "Kronecker compressive sensing," IEEE Transactions on Image Processing, vol. 21, no. 2, pp. 494-504, 2012.

[30] J. A. Tropp, M. B. Wakin, M. F. Duarte et al., "Random filters for compressive sampling and reconstruction," in Proceedings of the International Conference on Acoustics, Speech and Signal Processing, vol. 3, pp. 67-70, May 2006.

[31] M. Grant and S. Boyd, "CVX: Matlab software for disciplined convex programming," Version 2.1, 2014, http://cvxr.com/cvx. 


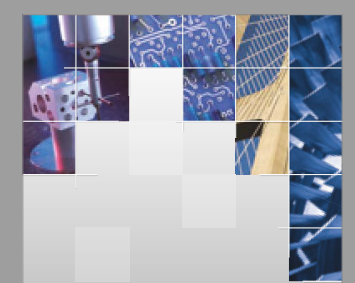

\section{Enfincering}
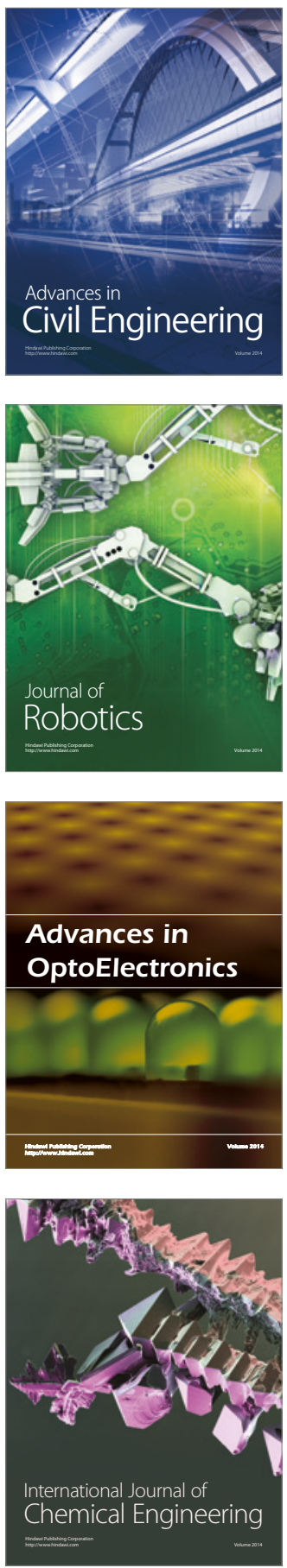

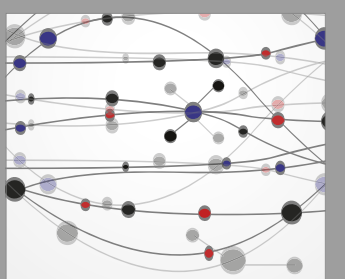

The Scientific World Journal

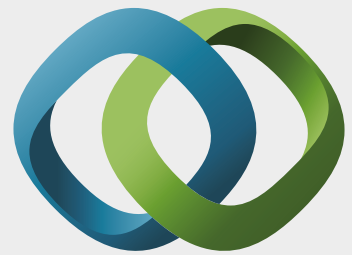

\section{Hindawi}

Submit your manuscripts at

https://www.hindawi.com
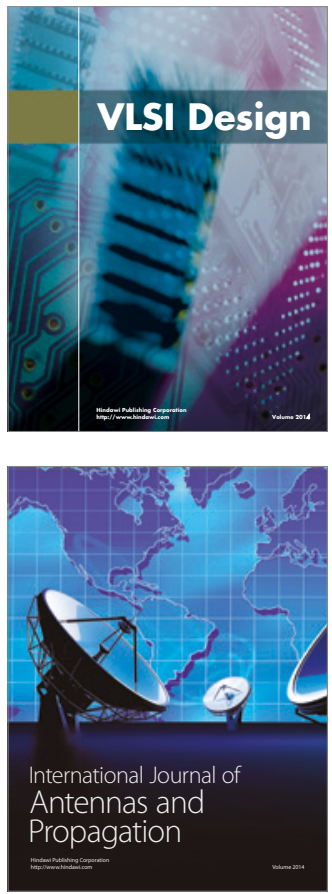

\section{Rotating}

Machinery
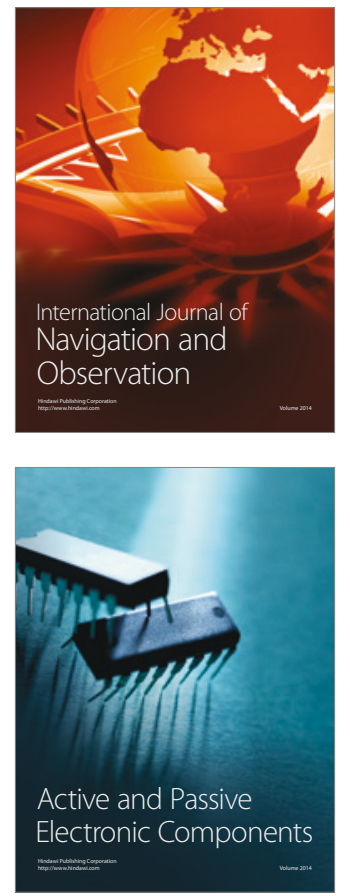
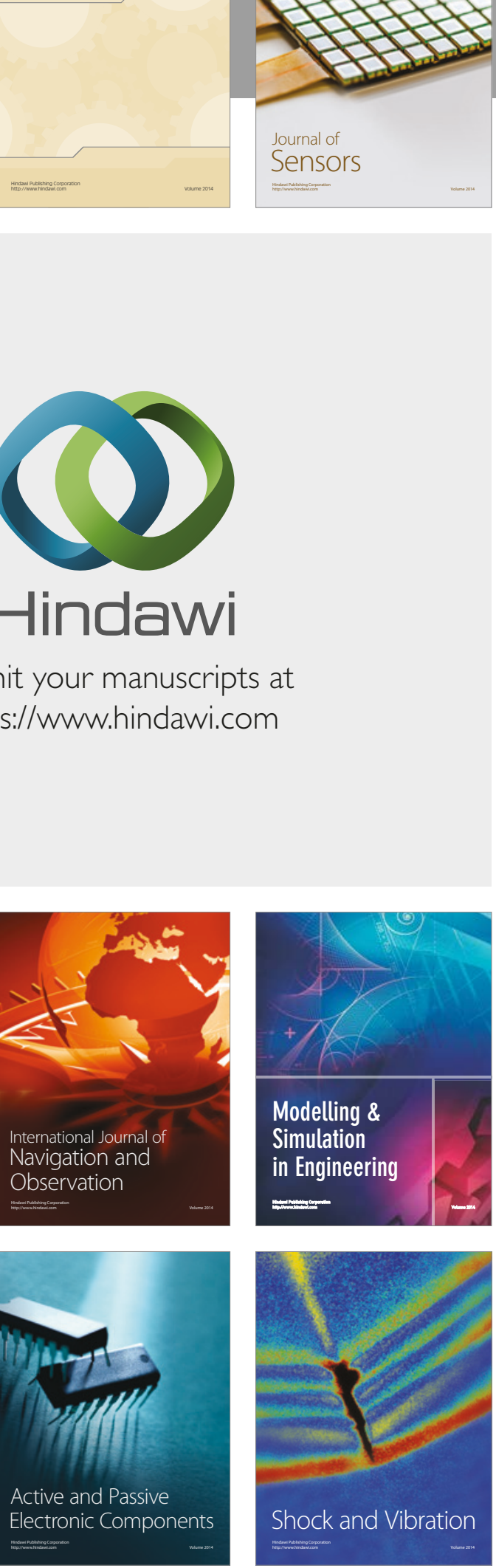
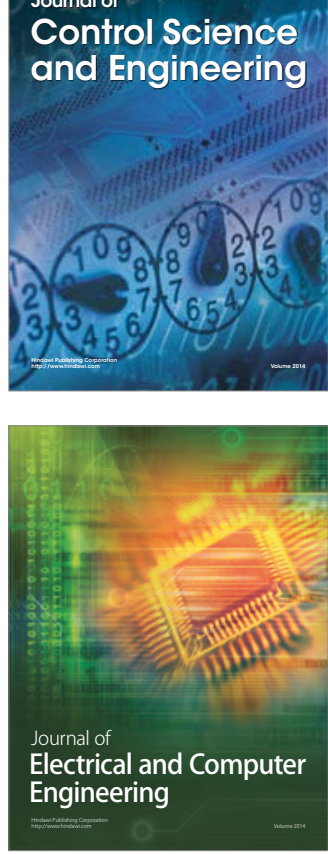

Distributed

Journal of

Control Science

and Engineering
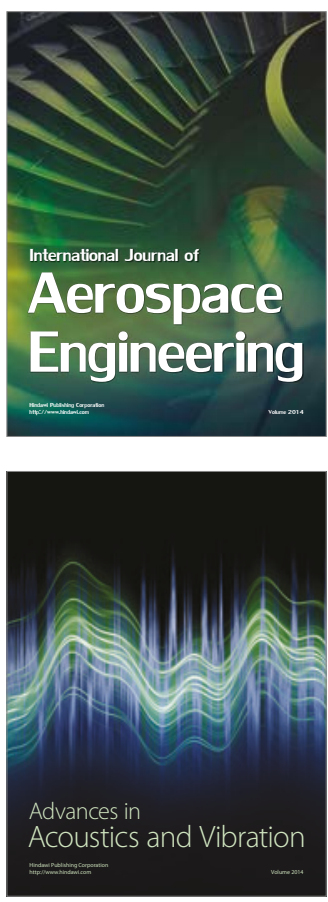

Sensor Networks 